ANNALS OF

CLINICAL

NEUROPHYSIOLOGY

\title{
Autonomic instability in severe tetanus: a case report
}

\author{
Seung Won Seo ${ }^{1}$, Jaewon Lee', Bong-Goo Yoo ${ }^{1}$, Jehun Kim² ${ }^{2}$ So-Young Huh ${ }^{1}$ \\ 'Department of Neurology, Kosin University College of Medicine, Busan, Korea \\ ${ }^{2}$ Department of Pulmonary and Critical Care Medicine, Kosin University College of Medicine, Busan, Korea
}

Received: June 4, 2021

Revised: July 15, 2021

Accepted: July 21, 2021

\section{Correspondence to}

\section{So-Young Huh}

Department of Neurology, Kosin University College of Medicine, 262 Gamchun-ro, Seogu, Busan 49267, Korea

Tel: +82-51-990-6461

Fax: +82-51-990-3077

E-mail: caccu@naver.com

\section{ORCID}

\section{Seung Won Seo}

https://orcid.org/0000-0002-9486-0161

Jaewon Lee

https://orcid.org/0000-0002-5649-7074

Bong-Goo Yoo

https://orcid.org/0000-0001-8813-7543

Jehun Kim

https://orcid.org/0000-0002-2594-0256

\section{So-Young Huh}

https://orcid.org/0000-0002-3309-6155

Tetanus is an infectious disease of the nervous system caused by Clostridium tetani, and is characterized by tonic muscle contractions, painful spasms, and autonomic dysfunction due to disinhibition of the anterior horn cells and autonomic neurons. ${ }^{1}$

Tetanus toxins transfer in a retrograde direction from the motor neurons to the spinal cord and into the inhibitory interneurons, interrupting exocytosis and causing inactivation of the inhibitory spinal interneurons. ${ }^{1}$ This increases excitation of the motor, cranial, and autonomic peripheral nerves. Reportedly $96-97.7 \%$ of tetanus patients experience lockjaw, $^{1}$ and another relatively common physical feature of tetanus is opisthotonos. ${ }^{1}$ Autonomic instability and sudden cardiac death associated with autonomic dysfunction have been reported in $32-62 \%$ and $11 \%$ of patients, respectively. 2,3

Tetanus is uncommon in South Korea due to the extensive nationwide childhood immunization program, but it remains life-threatening condition. Furthermore, autonomic instability is associated with severe tetanus and can result in poor prognoses for patients with mild to moderate symptoms. ${ }^{2}$ We report a case of tetanus with cardiovascular instability and review its autonomic dysfunction treatment. 


\section{CASE}

We report a 62-year-old female from our center who experienced intense pain and rigidity in the neck and jaw muscles, dysarthria, and difficulty in opening the mouth. The patient had a recent history of surgical debridement for an open left ankle fracture caused by a fall outside, followed by open reduction and internal fixation. Five days following the intervention, the patient developed a tremulous voice, swallowing difficulties, jaw pain, and neck stiffness, after which she was referred to our center. A further examination revealed that the patient was alert but had difficulty in articulation due to lockjaw. Hyperhidrosis and high blood pressure $(170 / 110 \mathrm{mmHg})$ were also observed. There were no abnormal movements or sensory symptoms at the time of admission. The patient was not vaccinated against tetanus after the trauma. Due to their previous open ankle fracture and clinical findings, the patient was diagnosed with tetanus, and we initiated treatment by administering intramuscular human tetanus immunoglobulin (5,000 units), tetanus-diphtheria vaccination, and metronidazole $(0.5 \mathrm{~g}$ every 6 hours). We also administered succinylcholine $(100 \mathrm{mg})$ to allow the mouth to open, and the patient was successfully intubated. The patient was kept on a mechanical ventilator in the intensive care unit (ICU) and was given midazolam (5-15 mg/hour) and magnesium sulfate (1 g/day). On the following day, changing the position of the patient and the wound dressing induced severe neck muscle spasms, high blood pressure, and sinus tachycardia of 140 beats/minute. The patient also developed a fever $\left(39^{\circ} \mathrm{C}\right)$ that could not be controlled by administering nonsteroidal anti-inflammatory drugs and broad-spectrum antibiotics. Myoclonic movement in the left leg was observed in the patient despite complete sedation. We administered vecuronium bromide (2-3 mg/ hour) to control the abnormal movements, which was effective in controlling both the muscle spasms and high fever. However, her systolic blood pressure (SBP) continued to fluctuate within the range of $40-250 \mathrm{mmHg}$, and her heart rate ranged from 50 to 120 beats/minute. The blood pressure fluctuated spontaneously, although the muscle spasms were inhibited. Intravenous nicardipine and labetalol were also administered to treat the severe hypertension (SBP > $200 \mathrm{mmHg}$ ). An excessive response to antihypertensive agents occurred, which decreased their blood pressure. The vasopressor agent and extensive hydration did not immediately affect hypotension. We intermittently administered norepinephrine and epinephrine to prevent a hypotensive attack. The target mean arterial pressure during vasopressor use is $65 \mathrm{mmHg}$. Despite this management, blood pressure fluctuations were observed for about 6 weeks when the vasopressor agents were administered for about 3 weeks (Fig. 1). Cardiovascular instability was most severe during the first 2 weeks after symptom onset. The blood pressure gradually stabilized, and consciousness was regained after discontin-

Variation of blood pressure

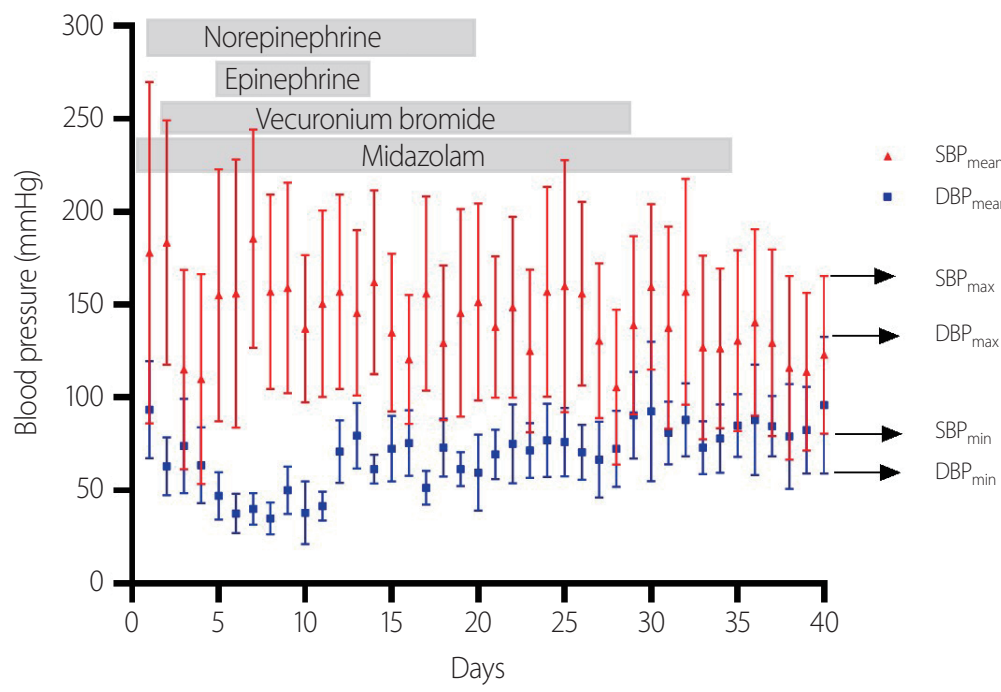

Fig. 1. Schematic diagram of changes in the blood pressure of the patient, the period of medical treatment, and the maximum (max), minimum (min), and mean arterial blood pressures over a 40-day period. Blood pressure fluctuation persisted despite 3 weeks of treatment and decreased gradually thereafter. SBP, systolic blood pressure; DBP, diastolic blood pressure. 
uing the sedatives. After 8 weeks, the patient experienced generalized muscle stiffness, but had recovered sufficiently to be able to open the mouth and eat. The patient was eventually transferred out of our ICU and referred to another hospital.

\section{DISCUSSION}

The Korea Disease Control and Prevention Agency estimates that tetanus occurs in 0.03-0.07 per 100,000 people each year, and nine deaths occurred between 2011 and 2019. ${ }^{4}$ The decline in tetanus incidence has largely resulted from the nationwide childhood vaccination program, which was originally implemented in 1956. However, the age of the present case suggests that they were not included in the nationwide childhood vaccine coverage.

Autonomic dysfunction presents with labile or persistent hypertension or hypotension, persistent sinus tachycardia, tachyarrhythmia, or bradycardia alternating with tachycardia, and sweating, or even fever in severe cases. ${ }^{1,2}$ These symptoms tend to become more prominent at 2 weeks after the onset of the disease, and the prognosis is often poor. ${ }^{1,2} \mathrm{Al}$ though autonomic dysfunction pathogenesis is unclear, the tetanus toxin prevents the synaptic vesicles from fusing with the cell membrane by cleaving synaptobrevin. This inhibits inhibitory interneuron discharge release, causing sympathetic hyperactivity and the excessive secretion of catecholamines. ${ }^{5}$ Motor neuron overexcitation also causes excessive acetylcholine secretion, which leads to parasympathetic overactivity. Autonomic dysfunction can therefore present with both sympathetic and parasympathetic overactivity. ${ }^{6}$

For tetanus-related airway management, it crucial to administer human tetanus immunoglobulin (500 or 3,000-6,000 units), a tetanus toxoid vaccination, and wound debridement. ' Sedation is essential for reducing any autonomic disturbance during ventilator support. Benzodiazepines augment g-aminobutyric acid (GABA) agonism by modulating the GABA-A receptor, thereby facilitating presynaptic inhibition in the brain and spinal cord. Benzodiazepines including diazepam and midazolam have been widely used to manage tetanus. ${ }^{7}$ However, diazepam requires a long recovery time, and its administration needs to be tapered slowly over weeks. Therefore, midazolam, which is a relatively short-act- ing benzodiazepine, is theoretically more effective than diazepam. ${ }^{8}$ However, there are limited data on the efficacy of midazolam.

Basal catecholamine concentrations, especially that of norepinephrine, increases about 10-fold, resulting in sympathetic storming. ${ }^{6,7}$ Various drugs with antiadrenergic activity including $a-, \beta-$, and combined $a-/ \beta$-adrenergic blockers have been used to manage this autonomic instability. However, some reports suggest that sudden death is associated with $\beta$-antagonists. ${ }^{7,8}$ The probable underlying mechanisms of these cases include sudden decreases in catecholamine concentration, negative inotropism, vasoconstriction, and unrestricted parasympathetic activity. ${ }^{6,7}$ Labetalol was introduced as a potential alternative, and reported some success. ${ }^{9}$ However, a case study on labetalol administration (via nasogastric tube at 150-800 mg/day, boluses of 5-75 mg, or infusion at 5-10 mg/hour) demonstrated similar issues to $\beta$-adrenergic blockers. ${ }^{10}$ Our patient also experienced sustained hypotension after the intravenous administration of $2.5 \mathrm{mg}$ of labetalol. Antisympathomimetic drugs should therefore only be considered when uncontrolled sympathetic overactivity is present despite adequate sedation. Esmolol (a short-acting $\beta$-blocker) and clonidine (a selective, partial a-adrenergic agonist) have been reported as promising alternatives, but more-robust evidence still needs to be obtained. $^{7.8}$

Magnesium sulfate, which is another adjunctive treatment for severe tetanus, is a calcium antagonist that induces vasodilation and presynaptic neuromuscular blockages, and prevents catecholamine release from the presynaptic neurons. ${ }^{8}$ Neuromuscular blockers are considered if muscle spasms continue despite administering benzodiazepines; for example, vecuronium is a short-acting neuromuscular blocker with minimal cardiovascular effects. ${ }^{8}$ In our patient, vecuronium administration effectively reduced both the myoclonic seizure-like movement and the uncontrolled high fever. Neuromuscular blockers are therefore recommended when preceded by adequate sedatives and analgesics to control muscle spasms in patients with severe tetanus.

Severe autonomic dysfunction associated with tetanus can be life-threatening, and so adequate immunization at an early stage of trauma is crucial. This case underlines the importance of managing cardiovascular instability, and its associated challenges. 


\section{Conflicts of Interest}

The authors have no conflicts to disclose.

\section{REFERENCES}

1. Berkowitz AL. Tetanus, botulism, and diphtheria. Continuum (Minneap Minn) 2018;24:1459-1488.

2. Wasay M, Khealani BA, Talati N, Shamsi R, Syed NA, Salahuddin N. Autonomic nervous system dysfunction predicts poor prognosis in patients with mild to moderate tetanus. BMC Neurol 2005;5:2.

3. Anuradha S. Tetanus in adults--a continuing problem: an analysis of 217 patients over 3 years from Delhi, India, with special emphasis on predictors of mortality. Med J Malaysia 2006;61:7-14.

4. Korea Disease Control and Prevention Agency. Infectious disease portal report [Internet]. Cheongju, Korea: Korea Disease Control and Prevention Agency; c2021 [accessed 2021 Mar 15]. Available from: http://www.kdca.go.kr/npt/biz/npp/ist/simple/simplePdStatsMain.do

5. Abrutyn E. Tetanus. In: Fauci AS, Braunwald E, Kasper DL, Hauser $\mathrm{SL}$, Longo DL, Jameson JL, eds. Harrison's principle's of internal medicine. 17th ed. New York: Mc Graw Hill Inc., 2008;898-900.

6. Spruyt GM, Van Den Heever T. The treatment of autonomic dysfunction in tetanus. South Afr J Crit Care 2017;33:28-31.

7. Farrar JJ, Yen LM, Cook T, Fairweather N, Binh N, Parry J, et al. Tetanus. J Neurol Neurosurg Psychiatry 2000;69:292-301.

8. Rodrigo C, Fernando D, Rajapakse S. Pharmacological management of tetanus: an evidence-based review. Crit Care 2014;18:217.

9. Dundee JW, Morrow WF. Labetalol in severe tetanus. Br Med J 1979;1:1121-1122.

10. Wesley AG, Hariparsad D, Pather M, Rocke DA. Labetalol in tetanus. The treatment of sympathetic nervous system overactivity. Anaesthesia 1983;38:243-249. 